\title{
INVESTIGATION OF ROLLED-UP EFFECT OF THE FORWARD- SWEPT 3-D WIND TURBINE BLADES
}

\author{
Sutrisno $^{1}$, Setyawan Bekti Wibowo ${ }^{2}$, Sigit Iswahyudi ${ }^{3}$ \\ ${ }^{l}$ Department of Mechanical and Industrial Engineering, Faculty of Engineering, Universitas Gadjah Mada, \\ Yogyakarta 55281, Indonesia \\ ${ }^{2}$ Department of Mechanical and Industrial Engineering, Faculty of Engineering, Universitas Gadjah Mada, \\ Yogyakarta 55281, Indonesia \& Department of Mechanical, Vocational College, Universitas Gadjah Mada, \\ Yogyakarta 55281, Indonesia \\ ${ }^{3}$ Department of Mechanical and Industrial Engineering, Faculty of Engineering, Universitas Gadjah Mada, \\ Yogyakarta 55281, Indonesia \& Department of Mechanical Engineering, Universitas Tidar, Magelang 56116, \\ Indonesia
}

\begin{abstract}
Noiseless aerodynamics of a rotor blade is a vital research objective towards environmentally friendly wind turbine technology and military advancement of helicopter blades. Blade planform of a wind turbine determines the limiting streamline that responsible for the aerodynamic characteristics. This paper focused on the aerodynamic flow visualization showing the flow pattern around forward swept horizontal axis wind turbine (HAWT) for different radius and different wind velocities. The experiments were conducted in a wind tunnel. Tuft patterns were used for flow visualization, and rope brake dynamometer was employed for measuring the performances in different wind speeds. It is found that the planforms can form 3-D folds streamline that show the detailed mechanism of rolling-up effect, and give different performances in the cases of different radius and wind speeds.
\end{abstract}

Keywords: Forward, 3-D wind turbine blades, Rolled-up vortex, 3-D folding effect, Limiting streamline

\section{INTRODUCTION}

The design and study of the HAWT blades initially paid little attention to rolled up vortices, the limiting streamline, 3-D folding streamlined effect, tip, and base flow angles of the turbine blades. At first, implementing blade element momentum (BEM) theory, the sectioned of HAWT blades were uniform as sliced independently with no spanwise exchange of momentum assumption. Blade construction and power tests were extensively conducted [1]-[4]. Substantial German and Russian scientists' contribution, from Joukowsky, Glauert had also been documented [5]. Centrifugal pumping effects and Coriolis force played a significant mechanism [6].

Singh and Ahmed have conducted power and blade construction test of a small rotor for low HAWT applications [7]. Bai et al., using numerical simulation, have performed an aerodynamic investigation and construction of $10 \mathrm{~kW}$ HAWT blade [2]. A revised BEM theory has been promoted to forecast the power of a constructed HAWT blade. There is a good agreement between the thrust and torque of the numerical simulation and the improved BEM theory. Velázquez et al. have described experimentation and design of a 1 MW HAWT [3]. Combination of any optimization algorithm and BEM is much proper to promote a more advanced formula. Plaza et al. have reported CFD and BEM comparison results for Mexico blade vortex dynamics [4]. Butterfield and Nelsen have reported stream demonstration experiments combination such as streamvisualization and fluid dynamic pressure measurement from a revolving HAWT blade employing a method of tuft [8], [9]. Some scientists [10], have reported an examination of an underlying implementation of a very little HAWT designed for multi-applications.

Some latest research on HAWT blade fluid dynamics were concentrated on simulation of the delay of the stall, the role of Coriolis and centrifugal forces due to spin which delays the point of separation [6]. The limiting streamlines of the surface help to see the blade tip and root flows generated by low and high-speed winds [11]. Dumitrescu and Cardos [12] concluded that the flow visualization was attached over the rotor, at low wind speed conditions. At about 0.17 to 0.31 of the radius of the rotor, the secondary stream reaches the fastest.

Several simulation studies on the 3-D effect of stall delays have been discussed. For low velocities HAWT, some researchers [12] have found that stall propagates from the base of the blade to the tip. Wu et al. have completed a study of the dynamic eddy arrangement during stall postponements [13]. Hu et al. have reported a study on the delay of the stall for HAWT [6]. Sicot et al. have proposed a method, on an investigation of stall-delay mechanisms, to determine the position of the separation point on the rotating 
blade [14]. Lee and Wu have reported a PIV study of stall delay experiment of a HAWT blade [15]. Yu et al. [11] have investigated on the flow properties of a HAWT rotating blade, at different wind velocities from $5 \mathrm{~m} / \mathrm{s}$ to $10 \mathrm{~m} / \mathrm{s}$. They shift from laminar, disturbed laminar, weak stall, half stall, stall to tip, and entirely stall.

The performance of 3-D HAWT blade models has been investigated focusing on stall suspension occurrence in relation with rearward and frontward swept of the HAWT blade styles. The stall delays have been recognized in 3-D HAWT frontward and rearward blade styles, in some occurrences there were, due to the RuV effects, resulted by triangle-hubs, as developed by front-edge vortices [16][18].

The purpose of this study is to investigate the performance of 3-D wind turbine blade models and verify the flow patterns using flow visualization. In this paper, the effect of forward swept of rotating wind turbine blades became the main focus of the current investigation.

\section{MATERIALS AND METHODS}

\subsection{Wind Turbine Design}

In this study, BEM procedures have been used to plan the geometries of the HAWT blades. The turbine had 3 blades and employed aerofoil NACA 4412 along with their spans. The pitch angles distributions and standardized chord lengths spanwise were approached using the BEM optimum spreading (Equations (1) and (2)), as displayed in Figure 1. The design used a $\lambda=3.65$. The performance of the plan is designated in the form of $\mathrm{Cp}$ as a function of $\lambda$, as shown in Figure 2. The experiment uses 2 types of HAWT blades of $\mathrm{R}=19 \mathrm{~cm}$ and $\mathrm{R}=14 \mathrm{~cm}$. Figure 3 displays $3-\mathrm{D}$ printed models that have a radius of $0.19 \mathrm{~m}$.

$$
\begin{aligned}
& \beta_{r}=\frac{2}{3} a \tan \left[\frac{1}{\lambda_{D}\left(\frac{r}{R}\right)}\right]-\alpha_{D}, \\
& \left(\frac{c}{R}\right)_{r}=\frac{16 \pi\left(\frac{r}{R}\right)_{r}}{C_{L, D}}\left\{\sin \left\{\frac{1}{3} a \tan \left[\frac{1}{\lambda_{D}\left(\frac{r}{R}\right)_{r}}\right]\right)\right\}^{2},
\end{aligned}
$$

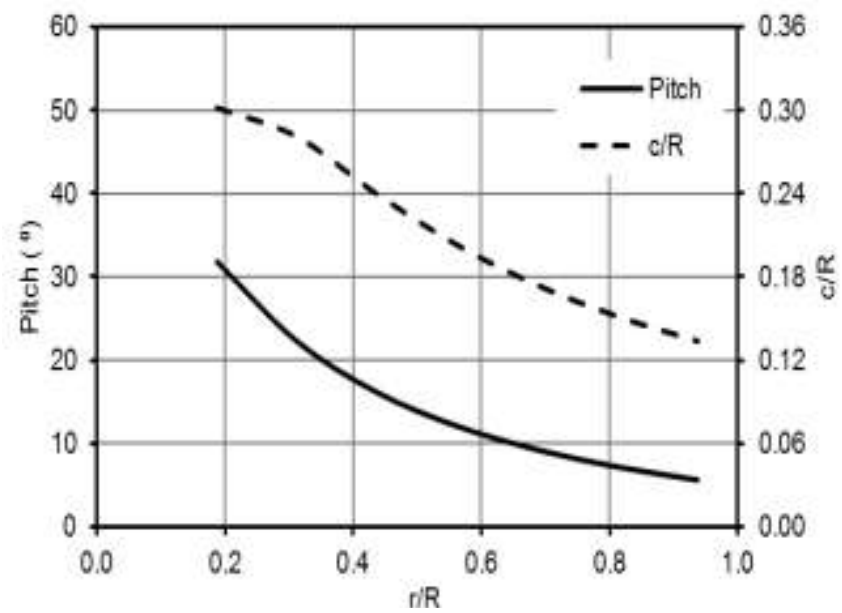

Fig 1: Designed pitch and normalized chord lengths spanwise [20]

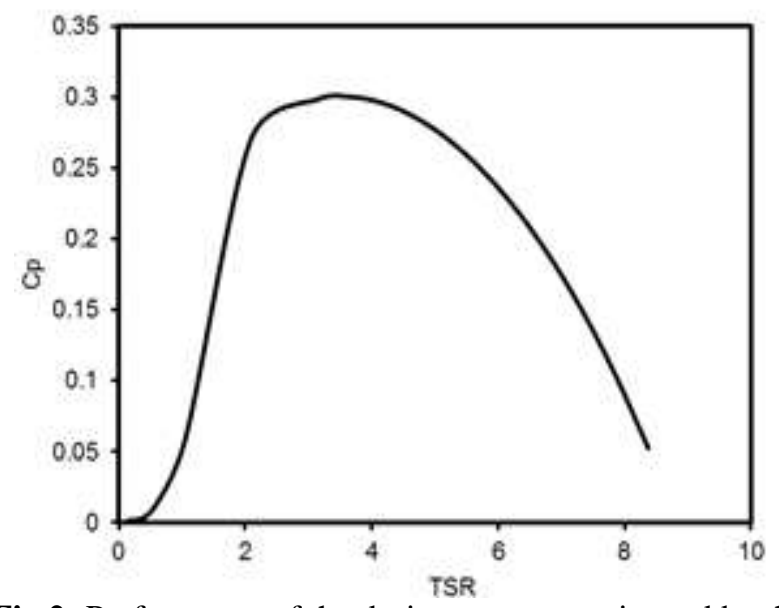

Fig 2: Performance of the design was approximated by the blade element method (BEM) [20]

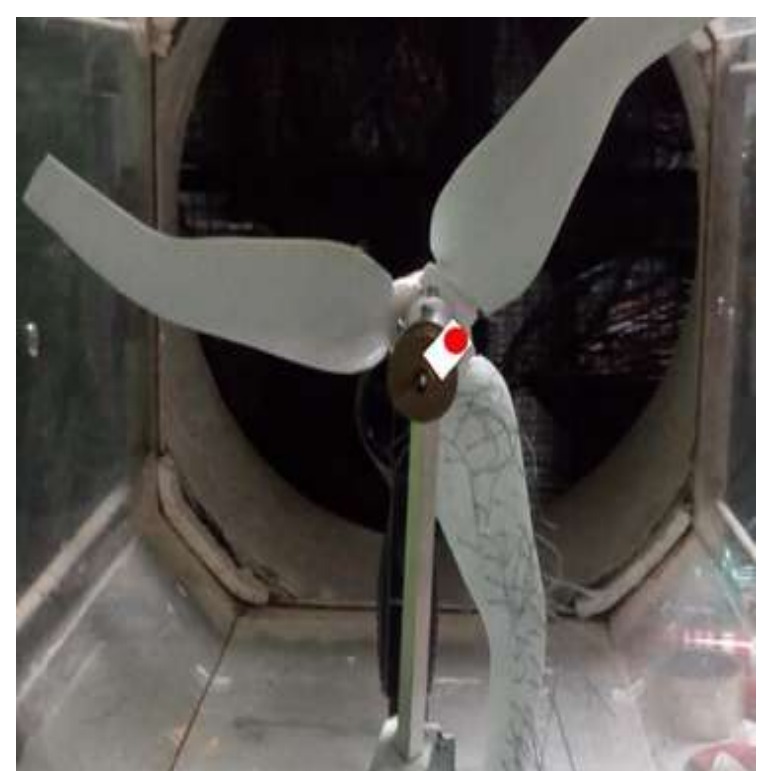

Fig 3: The 3-D printed models of radius $R=0.19 \mathrm{~m}$. It also displays the measurement of the RPM of the rotor 


\subsection{Experimental Methods}

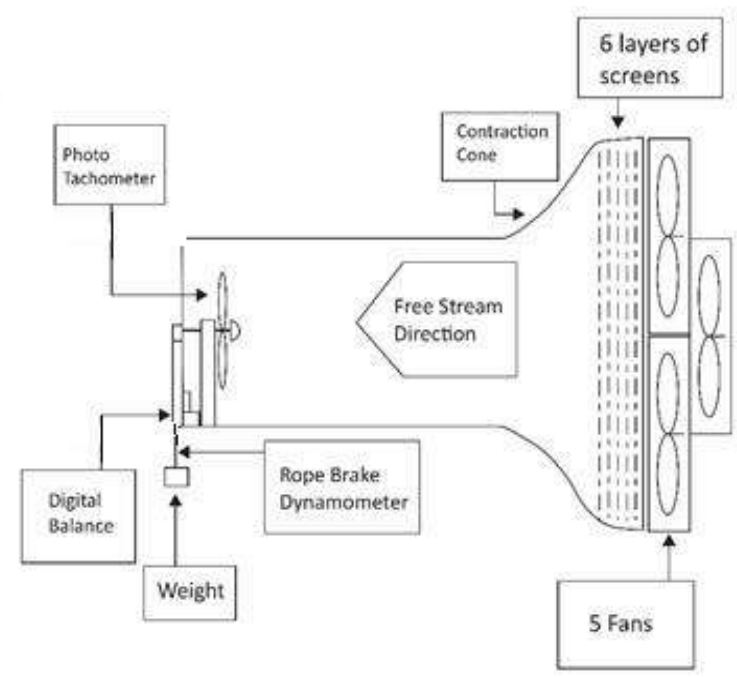

Fig 4: Test schematic in wind tunnel

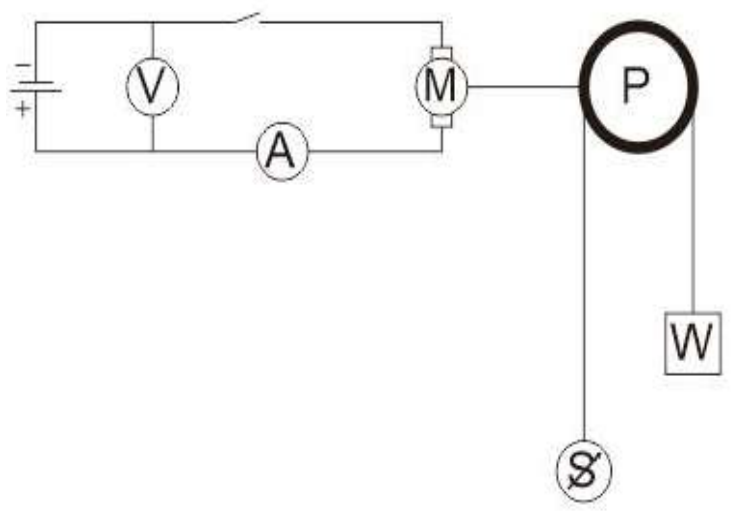

Fig 5: Rope brake dynamometer scheme

Experiment process was conducted in a wind tunnel. Fig. 4 shows the schematic elements of small-scale wind turbine measurement. The wind tunnel uses four blowers, and another one placed in the center to uniformize the wind input distribution. The air filter uses six arranged screen layers, the first two sparse screens, the second two medium sparse screens, and the third two tight screens, with the size of $20 \mathrm{~mm} \times 20 \mathrm{~mm}, 10 \mathrm{~mm} \times 10 \mathrm{~mm}$, and $5 \mathrm{~mm} \times 5 \mathrm{~mm}$. The uniform wind enters from the blower; the speed is made more uniform using the dissipation process that happens along the way to create quite a low turbulence wind. A welldesigned contraction cone produces better wind distribution. Conical contraction is designed with an electromagnetic theory so that the process of compressing the wind becomes as isentropic as possible. The wind tunnel modifiers consist of wind velocity, rotor speed, and the rotor torque. A rope brake dynamometer system measured the torque. The torque was not measured directly. A balancing weight $(W)$ was used to generate friction force on a pulley $D_{p}$ in diameter. The torque values were calculated from the difference between balancing weight and the values shown in digital balance $(S)$ by Eq. (3) [19].

$$
\tau=\frac{D_{p}}{2}(W-S)
$$

In order to know the efficiency of HAWT conversion into the rotation power, a tuft flow visualization method was applied to study the flow surrounding the forward HAWT blade models. The test scrutinized the flow pattern triggered by the blade actions.

\subsection{Rope Brake Dynamometer}

The dynamometer is an instrument for measuring power on a rotating shaft by measuring friction resistance or torsional friction. Due to the difficulty in measuring the friction, the rope brake dynamometer is much more reliable than a Prony-type brake system. Rope brake dynamometer, as it was assessed in [20]. This friction force is obtained by applying to brake. Rope brake dynamometer is one type of energy absorption type dynamometer. The way it works uses the braking principle between the rope and the pulley surface. In Fig. 5 consists of a shaft using a pulley and wrapped around a rope. One end of the rope is tied with a spring balance to read the string tension when the shaft to be measured rotates and one of them is tied to a load that can be varied. The frictional force between the rope and pulley must be determined to find out the amount of power produced by the shaft, so that torque is obtained in equation (3) [21], and the power generated by the shaft in equation (4) follows

$$
p=(W-S) \frac{D_{P}}{2} \frac{2 \pi N}{60}
$$

with $\mathrm{p}$ is the force generated by the shaft, W: Load $(\mathrm{N}), \mathrm{S}$ : The legible spring balance (N), DP: Pulley diameter (m), N: Shaft rotation (RPM) the tension of the rope in the spring balance and the load on equation (5) [22] below

$$
\frac{S}{W}=e^{\mu \theta}
$$

with $S$ : String tension tied to the mounting (N) $\mu$ : Strap coefficient $\theta$ : The contact angle between the rope and the pulley (rad)

\section{RESULTS}

\subsection{Visualization of the Limiting Streamlined}

\section{Forward Wind Turbine Blade}

Figure 6 and 7 show the visualization of the limiting streamline that occurs in the forward wind turbine blade of $R=19 \mathrm{~cm}$ and $R=14 \mathrm{~cm}$. In forward wind turbines blades up to $5.6 \mathrm{~m} / \mathrm{s}$ wind speed entirely stall phenomenon does not occur. This shows that the forward blade wind turbine can slow down the occurrence of the stall. 


\subsection{Performance Measurement of Forward}

\section{Wind Turbine Blade of Radius $19 \mathrm{~cm}$ and 14} cm

Figure 8, 9 and 10 show torques $(\mathrm{Nm})$, power $(\mathrm{Nm})$, and coefficient of power versus rotation per minutes (RPM) of forward wind turbine blades of $19 \mathrm{~cm}$ radius performance of different wind speeds. It is seen that when the wind velocity $\mathrm{V}$ goes down, as RPM drops, a) the torque of the wind turbine shaft goes down, and wind turbine power drops down. One can see that $C_{p}$ lines are almost similar for different wind speed.

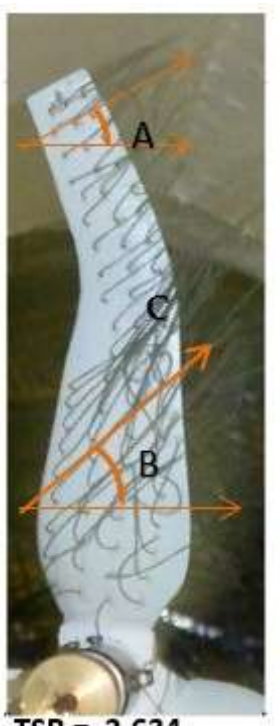

$\mathrm{TSR}=2.634$

370 RPM

$2.8 \mathrm{~m} / \mathrm{s}$

a) Disturbed Laminar

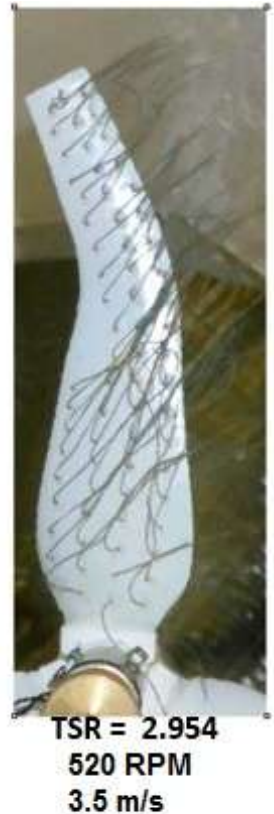

b) Disturbed Laminar c) Weak Stall

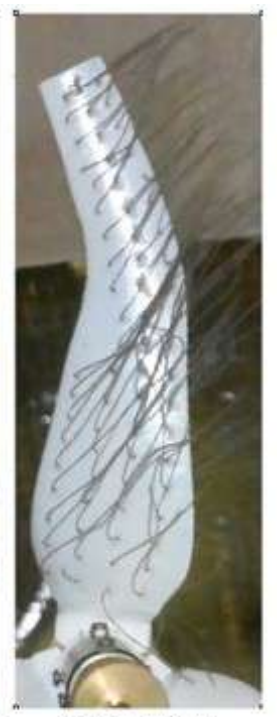

$\mathrm{TSR}=\mathbf{3 . 2 3 1}$

650 RPM

$4 \mathrm{~m} / \mathrm{s}$

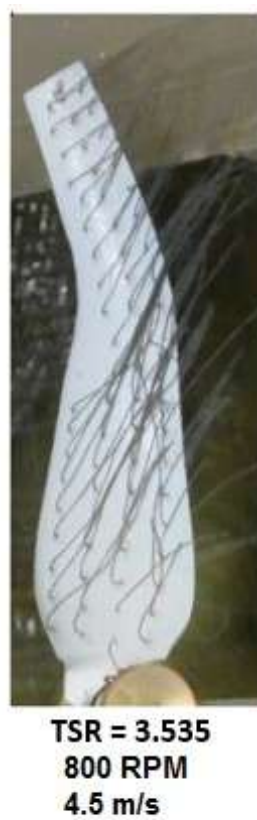

d) Weak Stall

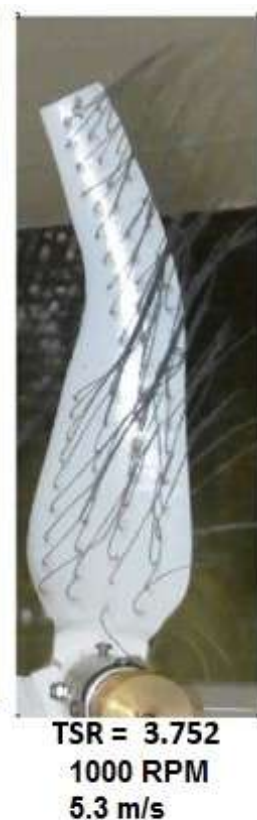

e) Half Stall

Fig 6: The limiting streamline visualization of forward wind turbine blade of radius $19 \mathrm{~cm}$.

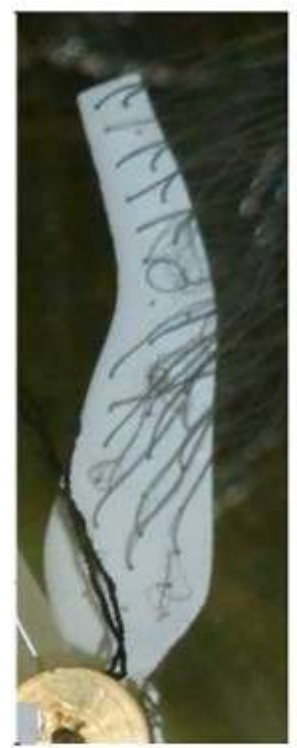

$\mathrm{TSR}=\mathbf{0 . 8 3 0}$

170 RPM

$3 \mathrm{~m} / \mathrm{s}$

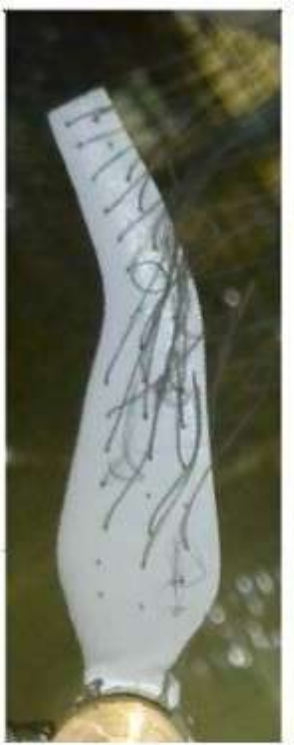

TSR $=1.663$

420 RPM

$3.7 \mathrm{~m} / \mathrm{s}$

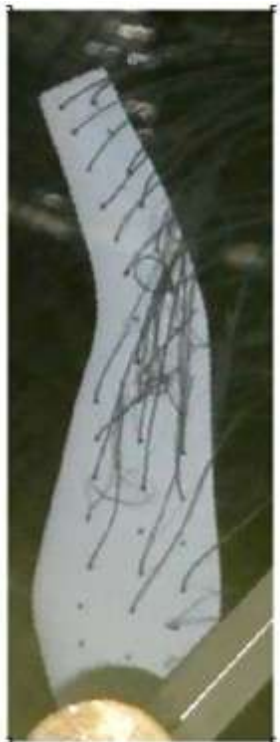

TSR $=1.746$ 560 RPM $4.7 \mathrm{~m} / \mathrm{s}$

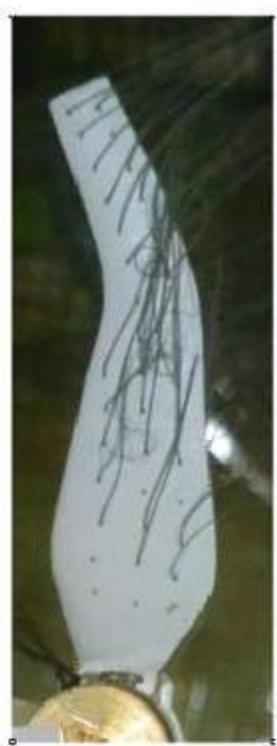

TSR $=1.781$ 620 RPM

$5.1 \mathrm{~m} / \mathrm{s}$

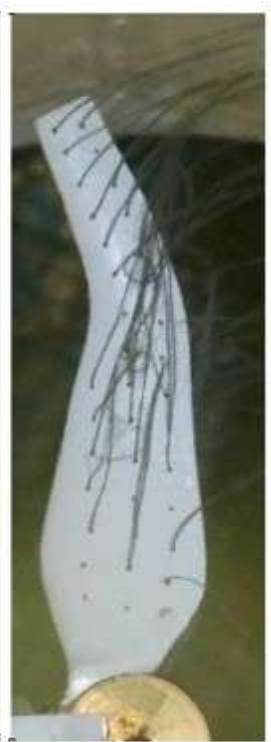

$\mathrm{TSR}=\mathbf{1 . 8 3 8}$

690 RPM

$5.5 \mathrm{~m} / \mathrm{s}$

e) Stall to Tip

Fig 7: The limiting streamline visualization of forward wind turbine blade of radius $14 \mathrm{~cm}$ 


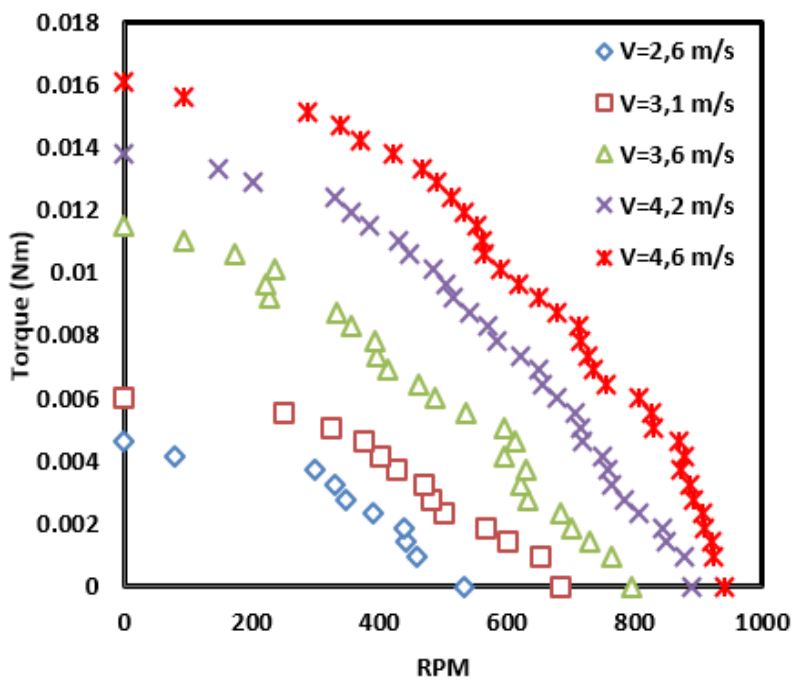

Fig 8: Torques (Nm) versus rotation per minutes (RPM) of forward wind turbine blades of $19 \mathrm{~cm}$ radius performance of different wind speeds

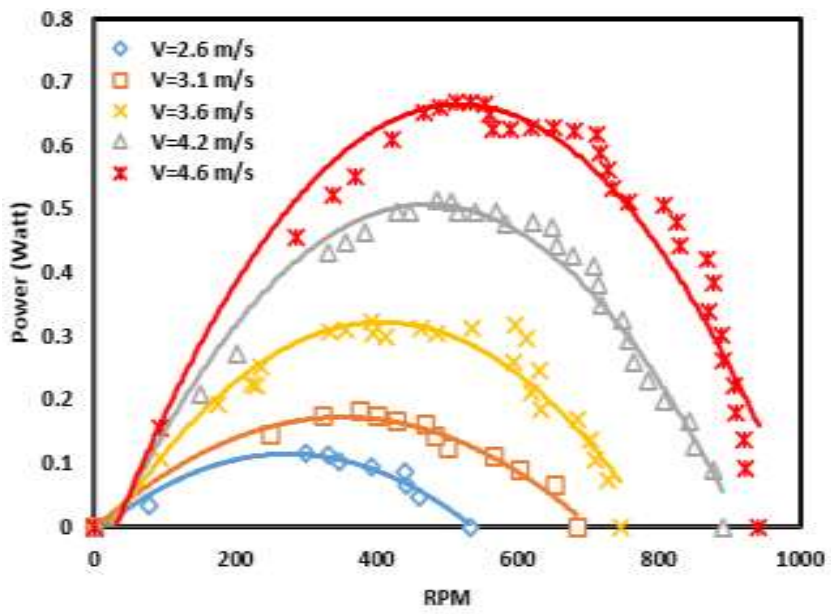

Fig 9: Power (Watt) versus rotation per minutes (RPM) of forward wind turbine blades of $19 \mathrm{~cm}$ radius performance of different wind speeds

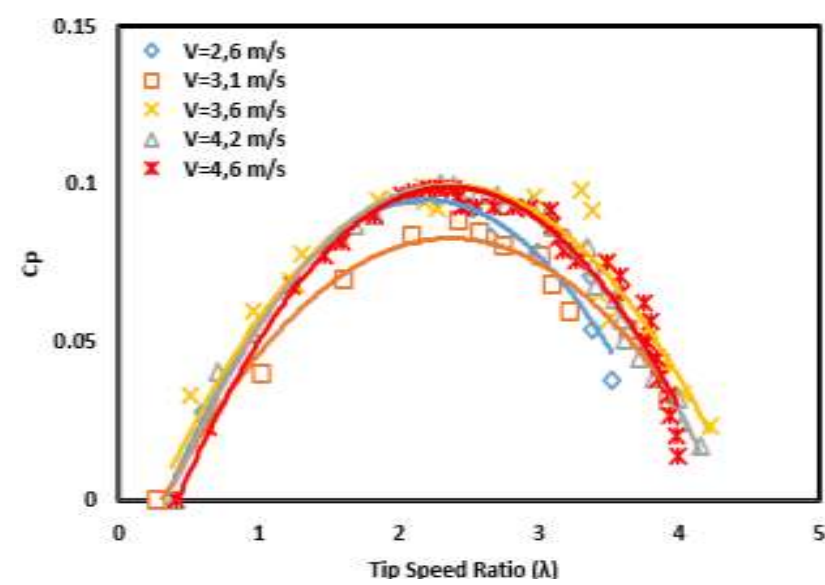

Fig 10: The coefficient of power $\left(C_{p}\right)$ versus rotation per minutes (RPM) of forward wind turbine blades of $19 \mathrm{~cm}$ radius performance of different wind speeds

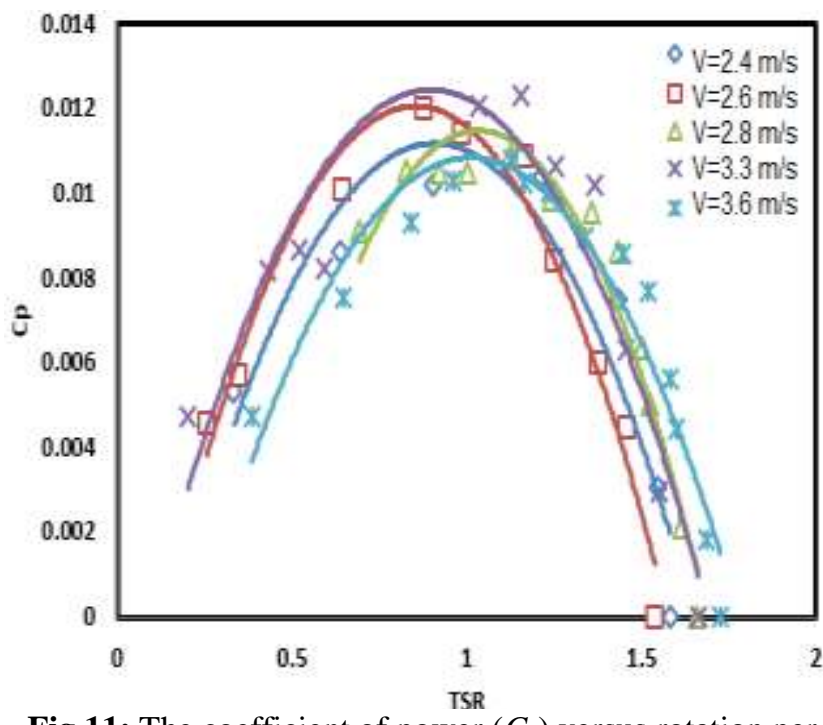

Fig 11: The coefficient of power $\left(C_{p}\right)$ versus rotation per minutes (RPM) of forward wind turbine blades of $14 \mathrm{~cm}$ radius performance of different wind speeds

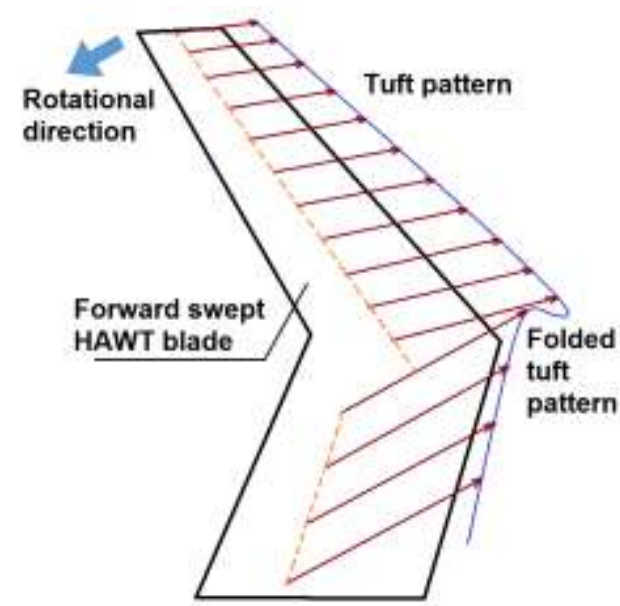

Fig 12: Aerodynamic pattern of the 3-D folds of the limiting streamlines of forward horizontal axis wind turbine blades

Fig. 11 shows the coefficient of power $\left(C_{p}\right)$ versus rotation per minutes of $14 \mathrm{~cm}$ radius performance of different wind speeds. One can recognize that the maximum coefficient of power $\left(C_{\text {pmax }}\right)$ of wind turbines with $19 \mathrm{~cm}$ radius at $\lambda=2.5$, is almost 8 times much stronger than the maximum coefficient of power $\left(C p_{\max }\right)$ of wind turbines with $14 \mathrm{~cm}$ radius at $\lambda=0.95$.

\section{DISCUSSION}

\subsection{Aerodynamics Flow Visualization of Wind} Turbine Blade

Flow visualization, in Fig. 6.a to 6.e shows that when V goes up, by that time RPM increases, $\lambda$ would go up. The limiting streamline, shown by the tuft pattern, indicates the flow angle $\mathrm{A}$ at the blade tip is smaller than the flow angle $\mathrm{B}$ at the base of the blade, and the rolled-up vortex creates a folded tuft sector or folded line $\mathrm{C}$. As V increases, the base 
flow angle increases with stronger speed, from Fig. 6.a to 6.e, the folded tuft line became tighter and the number of the fold lines increases. We call this process a 3-D folding effect of 3-D HAWT blade. The phenomenon shows the detailed mechanism of rolled-up effect of the 3-D folds of the limiting streamline of forward swept 3-D HAWT blade, as shown at Fig.12 They also demonstrate their effects on the RPM, TSR, and power of the wind turbines.

In Fig. 6.a the wind speed is $2 \mathrm{~m} / \mathrm{s}$ and the wind turbine rotation is 370 RPM. Judging from the tuff movement which shows that the position of the tuft is still reasonably perpendicular to the line of the wind turbine blade. This shows that laminar flow occurs on the surface of the forward wind turbine blade. In Fig. 6.b the conditions are $3.5 \mathrm{~m} / \mathrm{s}$ and 520 RPM. Appraising from the tuff movement which shows that the tuft position occurs with a slight flow disturbance at the root of the blade, this shows that disturbed laminar flow occurs on the surface of the forward turbine blade. In Fig. 6.c the conditions are $4 \mathrm{~m} / \mathrm{s}$ and 650 RPM. Considering the tuff movement which shows that the tuff has a flow disturbance at the root of the blade, started to spread to approximately one-quarter of the length of the blade, this shows that there is a weak stall flow on the surface of the blade. In Fig. 6.d the conditions are $4.5 \mathrm{~m} / \mathrm{s}$ and 800 RPM. Judging from the tuff movement which shows that it has a flow disturbance in the root of the blade and spreads to approximately half of the length of the blade. This shows that there is a half stall flow on the surface of the blade. In Fig. 6.e the conditions are $5.3 \mathrm{~m} / \mathrm{s}$ and 1000 RPM. Appraising from the tuff movement which shows that the tuff has a flow disturbance at the root of the wind turbine blade and spreads to approximately $3 / 4$ of the length of the wind turbine blade. This shows that stall to tip flow occurs on the surface of the forward wind turbine blade. Fig. 6 shows the visualization of the limiting streamlined that occurs in the forward wind turbine blade. In wind turbines, forward blades up to $5.3 \mathrm{~m} / \mathrm{s}$ wind speed do not occur entirely stall phenomenon. This shows that the forward blade wind turbine has the ability to slow down the occurrence of the stall.

It can be seen in Fig. 6 and 7, that when TSR goes higher while NR is constant but $V$ is low, then the tuft pattern is dragged by centrifugal force. When TSR is lower, then the tuft pattern is dragged by the wind. There are 2 sectors on the blade, the tip sector and the base sector. At small radius, $R=0.14 \mathrm{~m}$, compared with $R=0.19 \mathrm{~m}$, the effect of wind velocity is more dominant, as RPM effect is weak. We found that when $\mathrm{V}$ is $3 \mathrm{~m} / \mathrm{s}$ the blade pattern of $R=0.14 \mathrm{~m}$, is more laminar than the blade pattern of $R=0.19 \mathrm{~m}$ when, $V$ is $2 \mathrm{~m} / \mathrm{s}$.

\subsection{Aerodynamics Performance of Wind Turbine Blade}

It is shown in Fig. 8, 9, 10, and 11 the torque versus rotation per minutes of forward HAWT blades of $19 \mathrm{~cm}$ radius performance at different wind speeds. It can be seen, in Fig. 9. The torque increases as the wind speed rises and as the RPM reduces with constant wind velocities. Fig. 10 shows that the power increases as the wind speed rises and after the peak power, the RPM reduces with constant wind velocities. The graphs of the coefficient of power $\left(C_{p}\right)$ with respect to RPM of forward wind turbine blades of $14 \mathrm{~cm}$ and $19 \mathrm{~cm}$ radius performance were shown in Fig.10 and 11, for different wind speeds. The value of $C_{p}$ does not change so much for different wind speeds. And the $C_{p}$ drops significantly as the diameter reduces.

\section{CONCLUSION}

In this paper, we investigated the vortex dynamic characteristic of the flow surround the 3-D forward wind turbine blades in a wind tunnel. We employed a rope brake dynamometer system to measure the torque and tuft visualization method to explore the flow adjacent to the blades. The tunnel employed blowers with uniformized input distribution. The air filter used arranged screen layers, with its dissipation process along the way created wind with a quite low turbulence. It also employed a contraction cone, designed with electromagnetic theory, so that the process of compressing the wind becomes as isentropic as possible. A rope brake dynamometer system measured the torque, using a balancing weight to generate friction force on a pulley.

The system displays the wind speeds effect on the 3-D HAWT blade, on the torque, power, TSR, and $C_{p}$ of the wind turbines. Flow visualization shows that when $V$ goes up, at the same time the RPM increases, TSR will go up, the torque and the power increase as the wind speed rises and after the peak power, the RPM reduces with constant wind velocities.

As the wind speed and the wind turbine rotation increase, the tuff movement step by step shows the advancement from the disturbed laminar flow, weak stall flow, half stall flow and stall to the tip flow. The limiting streamline shows the rolled-up vortex which creates a folded tuft line. One could observe also the detailed mechanism of rolled-up effect of the 3-D folds of the limiting streamline of forward swept 3D HAWT blade. As the wind speed increases, the 3-D folding effect of 3-D HAWT blade increases. Then the stream angle increases with vigorous speed. When the folded tuft line becomes tighter, the fold line number increases. It shows the detailed rolled-up effects. The limiting streamline, shown by the tuft pattern, indicate the flow angle at the tip is smaller than the flow angle at the base of the blade, the rolled-up vortex creates a folded tuft line.

In the next step, it is hoped that one would be able to investigate more about the vortex dynamic characteristic of the flow surround the 3-D backward and combination of several geometric forms of leading edge and trailing edge of turbine blades. Therefore, it can be designed more turbine blades that laminarize the flow from leading edge to trailing 
edge which finally evokes noiseless turbine blades with high effectiveness, for HAWT and helicopter propeller blades.

\section{NOMENCLATURE}

$V \quad=$ the air velocity $(\mathrm{m} / \mathrm{s})$

$\tau \quad=$ the torque $(\mathrm{Nm})$

$N, \mathrm{RPM}=$ the frequency of the rotor (rotation/minutes)

$\lambda$, TSR $=$ tip speed ratio

$R \quad=$ blade outer radius $(\mathrm{m})$

$P \quad=$ output power of wind turbine $(\mathrm{W})$

$C_{p} \quad=$ coefficient of performance

$\rho \quad=$ air density $(\mathrm{kg} / \mathrm{m} 3)$

$D_{p} \quad=$ pulley diameter $(\mathrm{m})$

$p \quad=$ force generated by shaft

$\mu \quad=$ strap coefficient

$\theta \quad=$ contact angle

\section{ACKNOWLEDGMENTS}

This study was funded by the Government of the Republic of Indonesia Department of Research Technology and Higher Education, PTUPT-2018, under the contract 1859/UN1/DITLIT/DIT-LIT/LT/2018.The authors would like to express genuine appreciation to Prof. Indarto for helpful discussion, and fruitful collaboration. We appreciate the assistance of our students Farhan, Daffa, and Wega, and the lab staff members, Ponimin and Wajiono, for giving their help in construction work and conducting data management, which we gratefully acknowledge.

\section{REFERENCES}

[1] R. K. Singh and M. Ra, "Blade design and performance testing of a small wind turbine rotor for low wind speed applications," vol. 50, pp. 812-819, 2013.

[2] C. J. Bai, F. B. Hsiao, M. H. Li, G. Y. Huang, and Y. J. Chen, "Design of $10 \mathrm{~kW}$ horizontal-axis wind turbine (HAWT) blade and aerodynamic investigation using numerical simulation," Procedia Eng., vol. 67, pp. 279-287, 2013

[3] M. T. Velázquez, M. Vega, D. Carmen, J. A. Francis, L. A. M. Pacheco, and G. T. Eslava, "Design and Experimentation of a $1 \mathrm{MW}$ Horizontal Axis Wind Turbine," vol. 2014, no. January, pp. 9-16, 2014

[4] B. Plaza, R. Bardera, and S. Visiedo, "Comparison of BEM and CFD Results for MEXICO Rotor Aerodynamics," Jnl. Wind Eng. Ind. Aerodyn., vol. 145, pp. 115-122, 2015

[5] V. L. Okulov, J. N. Sørensen, and D. H. Wood, "The rotor theories by Professor Joukowsky: Vortex theories," Prog. Aerosp. Sci., pp. 1-28, 2014

[6] D. Hu, O. Hua, and Z. Du, "A study on stall-delay for horizontal axis wind turbine," Renew. Energy, vol. 31, no. 6, pp. 821-836, 2006

[7] R. K. Singh and M. R. Ahmed, "Blade design and performance testing of a small wind turbine rotor for low wind speed applications," Renew. Energy, vol. 50, pp. 812-819, 2013

[8] C. P. Butterfield, "Aerodynamic pressure and flowvisualization measurement from a rotating wind turbine blade," 1988

[9] C. P. Butterfield and E. N. Nelsen, "Aerodynamic Testing of a Rotating Wind Turbine Blade," Proc. Sol., 1990

[10] H. Hirahara, M. Z. Hossain, and M. Kawahashi, "Testing basic performance of a very small wind turbine designed for multi-purposes," vol. 30, pp. 1279-1297, 2005

[11] G. Yu, X. Shen, X. Zhu, and Z. Du, "An insight into the separate fl ow and stall delay for HAWT," Renew. Energy, vol. 36, no. 1, pp. 69-76, 2011

[12] H. Dumitrescu and V. Cardoş, "Inboard Stall Delay Due to Rotation," in Fundamental and Advanced Topics in Wind Power, R. Carriveau, Ed. IntechOpen, 2011

[13] Y. Wu, H. Mun, and H. Tang, "A study of the energetic turbulence structures during stall delay," Int. J. Heat Fluid Flow, vol. 54, pp. 183-195, 2015

[14] C. Sicot, P. Devinant, S. Loyer, and J. Hureau, "Rotational and turbulence effects on a win turbine blade. Investigation of the stall mechanisms," J. Wind Eng. Ind. Aerodyn., vol. 96, pp. 1320-1331, 2008

[15] H. M. Lee and Y. Wu, "An experimental study of stall delay on the blade of a horizontal-axis wind turbine using tomographic particle image velocimetry," J. Wind Eng. Ind. Aerodyn., vol. 123, pp. 56-68, 2013

[16] J. M. Mcdonough, "Lectures in Elementary Fluid Dynamics : Physics , Mathematics and Applications," Analysis, p. 163, 2009

[17] I. Ã. Gursul, Z. Wang, and E. Vardaki, "Review of flow control mechanisms of leading-edge vortices," Prog. Aerosp. Sci., vol. 43, pp. 246-270, 2007

[18] R. C. Nelson and A. Pelletier, "The unsteady aerodynamics of slender wings and aircraft undergoing large amplitude maneuvers," Prog. Aerosp. Sci., vol. 39, no. 2-3, pp. 185-248, 2003

[19] C. Han and S. A. Kinnas, "Study on the Wake Shape behind a Wing in Ground Effect Using an Unsteady Discrete Vortex Panel Method," Open J. Fluid Dyn., vol. 3, no. December, pp. 261-265, 2013

[20] S. Sutrisno, S. Iswahyudi, and S. Wibowo, "Dimensional Analysis in Power Prediction of a Real Scale Wind Turbine Based on Wind Tunnel Torque Measurement of Small Scaled Models," Energies, vol. 11, no. 9, pp. 1-13, 2018

[21] J. S. Brar and R. K. Bansal, A Text Book of Theory of Machines. Laxmi Publications, 2004

[22] R. Budynas and K. Nisbett, Shigley's Mechanical Engineering Design. New York: McGraw-Hill, 2011 


\section{BIOGRAPHIES}

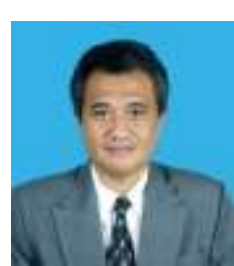

Sutrisno received a B.S. degree in Mechanical Engineering, Universitas Gadjah Mada, in 1980. M.S. and Ph.D. degrees from University Colorado, Boulder, the USA in 1985 and 1989. 